\title{
A comparison of abdominal muscles fatigue in women after vaginal birth, women after caesarean section, and a control group. A pilot study
}

\author{
DOI: https://doi.org/10.5114/pq.2020.100284
}

\author{
Natalia Kuciel', Justyna Mazurek ${ }^{1}$, Edyta Sutkowska', Karolina Biernat ${ }^{1}$, tukasz Pawik ${ }^{2}$ \\ ${ }^{1}$ Department of Rehabilitation, Faculty of Medicine, Wroclaw Medical University, Wroclaw, Poland \\ 2 Department of Physiotherapy in Motor System Dysfunctions, University School of Physical Education in Wroclaw, \\ Wroclaw, Poland
}

\begin{abstract}
Introduction. To investigate fatiguability of abdominal muscles in women after natural childbirth and caesarean section compared with a control group in the prone bridge (plank) test.

Methods. The study involved 11 women after natural childbirth, 8 women after caesarean section, and 8 women who had not been pregnant and had not given birth. After completing a personal questionnaire and qualification in a functional examination, the participants performed the prone bridge (plank) test. A 4-lead surface electromyograph was used for the study of rectus abdominis muscles and internal oblique/transversus abdominis muscles.

Results. There were no statistically significant differences between the right and left side of the body $(p<0.05)$. There were no differences in muscle fatigue parameters between the groups in the prone bridge test $(p<0.05)$.

Conclusions. Electromyographic parameters of abdominal muscles fatigue are similar in women after natural childbirth, women after caesarean section, and those who have not given birth. Caesarean section in asymptomatic women does not affect the deterioration of abdominal muscle strength in plank exercise.

Key words: abdominal wall, caesarean section, exercise, muscular fatigue, vaginal birth
\end{abstract}

\section{Introduction}

The abdominal wall plays an important role in maintaining posture, trunk and pelvic stability, respiration, trunk movement, and support of the abdominal viscera. The functional roles of the abdominal muscles during pregnancy appear to be similar to those in the nonpregnant state and include trunk movement, pelvic stabilization, and restraint of the abdominal contents. Many women continue, or even begin, abdominal exercise programs during their pregnancies. In addition, others are often encouraged to resume abdominal exercises shortly after delivery [1]. During and after pregnancy, many women experience an increase in the inter-recti abdominal muscle distance due to stretching and thinning of the linea alba, which occurs because of hormonal elastic changes of the connective tissue, mechanical changes placed on the abdominal wall by the growing foetus, and displacement of the abdominal organs [2]. This may result in altered trunk mechanics, impaired pelvic stability, and changed posture, which leave the lumbar spine and pelvis more vulnerable to injury [1]. After delivery, maternal abdominal muscle strength decreases and the fat ratio in the waist region increases [3].

For these reasons, lumbopelvic pain is common during pregnancy. Although a large proportion of women recover within 1 month after delivery, a substantial percentage (5-8.5\%) have persisting complaints even up to 2 years after delivery [4]. Biomechanical and anatomical studies have shown that transversely oriented muscles of the abdominal wall, especially the transverse abdominal muscle, in co-contraction with the pelvic floor, are the most suitable muscles to achieve compression in the lumbopelvic area and reduce movement in the sacroiliac joint [5, 6]. Fast et al. [7] reported that abdominal muscles during the third trimester of pregnancy were weakened relative to the abdominal muscles of nonpregnant control subjects. Other researchers, when assessing abdominal muscles strength at 6 and 12 weeks after delivery, observed no differences between women postpartum and nulliparous control subjects, despite evidence of incomplete musculoskeletal readaptation in the post-birth period [1].

Compared with delivery by caesarean section (CS), the morbidity rate of mothers after vaginal delivery (VD) is less, and their recovery period is shorter [8]. Physical activity is recommended for pregnant women because it plays an important role in reducing a potential risk of instrumental childbirth and CS [9]. Delivery by CS generally decreases damages to the pelvic floor muscles (affecting urinary incontinence) but it damages abdominal muscles and can cause intestinal obstruction, chronic pelvic girdle pain, infertility, or difficulties due to repeated surgery. CS causes damage to the abdominal muscle resulting from surgery, with the additional possibility of adhesion [8]. The knowledge of the abdominal muscles' morphological adaptations and their functional abilities, as well as of the relationship between muscle structural changes and functional ability is essential for the continued development of prenatal and postnatal physical therapy programs.

The objective of this study was to investigate fatiguability of abdominal muscles in women after VD and CS compared with a control group (CG) in the prone bridge (plank) test with the use of surface electromyography.

Correspondence address: Natalia Kuciel, Department of Rehabilitation, Faculty of Medicine, Wroclaw Medical University, ul. Borowska 213, 50-556 Wroclaw, Poland, e-mail: nataliakuciel@interia.pl 


\section{Subjects and methods}

\section{Participants}

Patients were recruited on a social networking site in a closed perinatal group, within 6-12 months after the birth. The study involved 11 women after VD, 8 women after CS, and 8 women who had not been pregnant and had not given birth (CG). The patients were informed about the examination procedure. The inclusion criteria in the group of women after VD were as follows: no pain in the lumbar, pelvic, or abdomen area; first pregnancy and childbirth without additional procedures such as ticks or vacuum; negative pain and mobility tests of the spine, hip, and knee joints; negative tests in physical examination (posterior pelvic pain provocation, long dorsal ligament test, Gaenslen test, Trendelenburg test); no diastasis recti abdominis according to the latest gynaecological examination. The inclusion criteria in the group of women after CS involved no pain in the lumbar, pelvic, or abdomen area; first pregnancy and childbirth; planned CS without complications; negative tests mentioned above in physical examination; no diastasis recti abdominis according to the latest gynaecological examination. The inclusion criteria in CG were no pain in the lumbar, pelvic, or abdomen area; no pregnancies and childbirths; negative tests in physical examination. The exclusion criteria for all groups were the following: disagreement on participation; lumbar or sacral pain; pelvic pain; abdominal pain; 2 or more deliveries; sudden CS; VD with additional procedures; positive tests in physical examination; problem with the proper performance of the motor task (plank).

\section{Questionnaire}

The participants completed a personal questionnaire concerning socio-demographic and clinical data: age, method of pregnancy termination, type of work, and information on the occurrence of lumbosacral pain and urinary incontinence. The measurement of height, body weight, and body mass index was performed and supplemented in the questionnaire by a physiotherapist examining the patient.

\section{Functional tests used in examination}

Functional tests were performed by a person taking measurements (physiotherapist) to exclude pain and mobility restrictions on the spine, hip, and knee joints (Table 1). The tests used in the examination were: spinal column mobility tests in the sagittal, frontal, and rotational plane in a standing position; passive hip joint mobility tests in the aforementioned planes; passive knee joint mobility test; posterior pelvic pain provocation tests; hip-lumbar ligament pain provocation test; Trendelenburg test; and Gaenslen test.

The examination was conducted by using a MyoTrace 400 4-lead surface electromyograph (sEMG) manufactured by Noraxon Company, USA. The analyses of the results and data were recorded in the MyoResearch Master Edition software. Paediatric Bio Lead-Lok electrodes (produced by Vermed, Lendersdorf, Germany) with a diameter of $30 \mathrm{~mm}$ with $\mathrm{Ag} /$ $\mathrm{AgCl}$ sensors were used in the study. Before applying the electrodes, the skin was cleansed with salicylic spirit to reduce its impedance. The cables were attached to the skin with a tape to minimize the movement of the electrodes. The electrodes were fixed during lying on the back and on both sides in the following sites:

1. Transverse abdominal/internal oblique muscle: $2 \mathrm{~cm}$ below the anterior superior iliac spine, in the lower medial direction [13].

2. Lower rectus abdominis muscle: middle point between the navel and pubic symphysis, $3 \mathrm{~cm}$ to the side [14].

The electrodes were attached at a distance of $2 \mathrm{~cm}$ from each other. The reference electrode was placed on the right iliac crest. Fast Fourier transform - unfiltered raw signal of sEMG analysed stepwise over the 15-second static plank contraction [15] - was used for fatigue analysis. The following parameters were analysed: slope (regression line), intercept values $(\mathrm{Hz})$, and mean frequency difference between first 3 and last 3 period values (\%).

\section{Prone bridge (plank) test}

The participants performed the prone bridge test (plank exercise). In the test, a mat with a marked point was needed to fix the eyes. The examined person took a position in the support on the forearms and toes. The eyesight was fixed on the point marked on the mat and the neck was in a neu-

Table 1. Functional tests in examination

\begin{tabular}{|c|c|}
\hline Test & Performance \\
\hline $\begin{array}{l}\text { Mobility of the spine in sagittal, frontal, } \\
\text { rotational plane }\end{array}$ & $\begin{array}{l}\text { Standing position, feet at hip width, hands along the trunk. Execution: flexion and extension } \\
\text { of the trunk; lateral bending to the right and left; rotation to the right and left [10] }\end{array}$ \\
\hline Mobility of the hips & $\begin{array}{l}\text { Supine position: passive examination of flexion, internal and external rotation, and abduction } \\
\text { and adduction of the hip. Prone position: passive hip straightening test [10] }\end{array}$ \\
\hline Mobility of the knees & $\begin{array}{l}\text { Supine position: passive examination of flexion and extension, internal and external rotation } \\
\text { of the knees [10] }\end{array}$ \\
\hline Posterior pelvic pain provocation test & $\begin{array}{l}\text { Supine position, hip bent up to } 90^{\circ} \text { on the test side. A gentle pressure is applied } \\
\text { to the bent knee along the long axis of the femur, while the pelvis is stabilized by the other } \\
\text { hand of the examiner on the contralateral frontal upper iliac spine [11] }\end{array}$ \\
\hline Long dorsal ligament test & $\begin{array}{l}\text { Lying on one side with the hips and knees bent. The test involves palpation } \\
\text { of the longitudinal dorsal ligament [11] }\end{array}$ \\
\hline Gaenslen test & $\begin{array}{l}\text { Supine position. Stabilization of the trunk by bending the opposite limb and stabilizing it } \\
\text { to the trunk. The tested limb, outside the couch, is positioned in hyperextension [12] }\end{array}$ \\
\hline Modified Trendelenburg test & Standing position. Lifting one leg bent in hip and knee joints into $90^{\circ}[11]$ \\
\hline
\end{tabular}

Surface electromyography 
tral position. The head, the spine, and the hips were aligned. The shoulders were above the elbows, forearms in the neutral position between pronation and supination. The feet were set at hip width. The participants maintained this position for 15 seconds. If, during the test, the patient changed the position of the pelvis (lifted or lowered from the alignment), the test was completed [16]. At the beginning, the patients performed an initial test to become familiarized with the procedure; afterwards, the prone bridge test was carried out 3 times (each test took 15 seconds). After the command 'up,' the patient lifted the trunk and held the test by 15 seconds until the command 'down'. The rest between the measurements was 30 seconds. The command was the same for all of the participants.

\section{Statistics}

The Statistica 10.0 program was used for statistical analysis. ANOVA with a post-hoc test served to evaluate the differences between the groups. The differences between the right and left side of the body were assessed with Student's $t$-test for dependent variables. Statistical significance was assumed at the level of $p<0.05$.

\section{Ethical approval}

The research related to human use has complied with all the relevant national regulations and institutional policies, has followed the tenets of the Declaration of Helsinki, and has been approved by the Bioethics Committee of Wroclaw Medical University (opinion No.: KB - 321/2018).

\section{Informed consent}

Informed consent to participate in the study and to process their personal data has been obtained from all individuals included in this study.

Table 2. Characteristics of the groups

\begin{tabular}{|l|c|c|c|c|}
\hline Characteristics & $\begin{array}{c}\text { Group 1 } \\
\text { mean (SD) }\end{array}$ & $\begin{array}{c}\text { Group 2 } \\
\text { mean (SD) }\end{array}$ & $\begin{array}{c}\text { Group 3 } \\
\text { mean (SD) }\end{array}$ & $\begin{array}{c}p \\
(\text { ANOVA) }\end{array}$ \\
\hline Age (years) & $28.09(3.3)$ & $31.62(2.77)$ & $25.12(4.67)$ & 0.005 \\
\hline Weight (kg) & $58.5(8.14)$ & $55.8(9.20)$ & $60.06(7.07)$ & 0.592 \\
\hline Height (m) & $1.66(0.05)$ & $1.65(0.06)$ & $1.70(0.06)$ & 0.121 \\
\hline BMI (kg/m²) & $21.11(2.23)$ & $20.39(2.57)$ & $20.76(2.91)$ & 0.825 \\
\hline
\end{tabular}

1 - group after vaginal delivery,

2 - group after caesarean section

3 - control group

$\mathrm{BMI}$ - body mass index

\section{Results}

The studied groups were not homogeneous in terms of age. Women in the CG were the youngest and women in the group after CS were the oldest (Table 2).

There were no statistically significant differences between the right and left side of the body (Table 3). Therefore, sEMG results for the right side of the body were used to analyse the differences between the groups.

There were no statistically significant differences in muscle fatigue parameters between the groups in the prone bridge test (Table 4), although the groups were not homogeneous in terms of age.

\section{Discussion}

After childbirth, women experience many problems with the locomotor system. Dysfunctions such as stress urinary incontinence, diastasis recti abdominis, or lumbar and pelvic girdle pain lower the quality of life and make it difficult for mothers to care about the newborn baby. The authors analysing the activity of abdominal muscles focus on the necessity to choose appropriate exercises in the context of the above mentioned problems [17]. Much attention has been paid in the literature to the problem of rehabilitation of diastasis recti abdominis and urinary incontinence, as well as the construction of a pneumatic cylinder which creates proper abdominal pressure and conditions to support the internal organs and maintains adequate stiffness in the lumbar and sacral section [18-21]. However, there are scarce data comparing the activity of abdominal muscles in women after VD and CS when none of the above mentioned ailments occur. It is difficult to relate the results of tests to specific dysfunctions in the group of women after CS if the parameters of muscles activity in painless women are not known.

Table 4. Differences between the groups in fast Fourier transform fatiguability parameters

\begin{tabular}{|l|c|c|}
\hline Parameters & $F$ & $p$ \\
\hline Slope RRA & 58643 & 0.56408 \\
\hline Slope RIO/TrA & 51066 & 0.606469 \\
\hline Intercept RRA & 1.81374 & 0.18468 \\
\hline Intercept RIO/TrA & 0.76982 & 0.474164 \\
\hline Difference RRA & 0.277705 & 0.759921 \\
\hline Difference RIO/TrA & 0.366444 & 0.697007 \\
\hline
\end{tabular}

RRA - right rectus abdominis, RIO/Tra - right internal oblique/ transversus abdominis; $F$-Friedman's ANOVA

Table 3. Differences between right and left side of the abdomen in fast Fourier transform fatiguability parameters in the tested groups

\begin{tabular}{|l|c|c|c|c|c|c|}
\hline \multirow{2}{*}{ Parameters } & \multicolumn{2}{|c|}{ Group 1 } & \multicolumn{2}{c|}{ Group 2 } & \multicolumn{2}{c|}{ Group 3 } \\
\cline { 2 - 7 } & $t$ & $p$ & $t$ & $p$ & $t$ & 0 \\
\hline Slope RA & -0.8077 & 0.4380 & -0.7289 & 0.4898 & 1.7806 & 0.1181 \\
\hline Slope IO/TrA & -0.5279 & 0.60904 & 1.5270 & 0.1708 & 0.1078 & 0.917172 \\
\hline Intercept RA & -0.4346 & 0.6730 & -0.0110 & 0.9915 & -1.6327 & 0.14655 \\
\hline Intercept IO/TrA & 0.9412 & 0.36879 & -1.1550 & 0.28609 & 0.0432 & 0.966741 \\
\hline Difference RA & -0.8993 & 0.389616 & -0.9443 & 0.37644 & 1.4942 & 0.17876 \\
\hline Difference IO/TRA & -0.4163 & 0.6859 & 1.0212 & 0.341139 & -0.3676 & 0.72400 \\
\hline
\end{tabular}

1 - group after vaginal delivery, 2 - group after caesarean section, 3 - control group, 
CS is currently performed in $40-50 \%$ of cases of women giving birth [22]. It is a procedure associated with a number of ailments, such as postoperative adhesions or scar endometriosis. Additionally, abdominal fascia incision may lead to altered motor control and worse stability of the lower spine by disturbing the anticipatory function of the transverse abdominal muscle $[23,24]$. Plank exercise was used in this study to determine muscle fatigue and differences between women after VD and CS. It has been applied as a reliable test to evaluate the performance of selected muscles in static conditions. In the study, the groups were heterogeneous in terms of age. Women after CS gave birth to their first child later than those in the group after VD. Nevertheless, the analysis of the test did not reveal any significant differences in the parameters of abdominal muscle fatigue. This may be due to a number of issues: 6 months is the recommended time to wait after CS for more intensive exercises of the abdominal muscles in order to obtain a full recovery of the abdominal wall [25]. In addition, the groups were examined without any pain, which could also affect the similar picture of the data. On the basis of the above results, it can be concluded that 6 months after the delivery, the delivery type should not affect the parameters of abdominal muscles fatigue, so the 15-second plank can be a valuable exercise to both evaluate the work of these muscles and strengthen abdominal muscles.

Pregnant women have numerous questions about the benefits and disadvantages of each type of birth [26]. There is a lack of studies to confirm the knowledge about the possible postnatal consequences for the core muscles, which may evoke fear of commencing fitness exercises [26, 27]. In addition, the pressure to return to the prenatal form as soon as possible is now widespread. Patients do not always know when they can start exercise or how intensive the physical effort should be to turn out beneficial for them [28, 29]. They fear that they will not be able to recover the abdominal muscle strength to the state before pregnancy or, on the contrary, they expect to return to full performance too quickly [30]. It is known that exercise in pregnancy may improve recovery of strength and endurance of the abdominal muscles after pregnancy and childbirth. Therefore, it seems so important to analyse the above topic in order to improve the conscious rehabilitation process enabling the recovery of strength and endurance of abdominal muscles after pregnancy and childbirth. An appropriately selected rate of convalescence adapted to the type of labour seems to be the key issue constituting the basis for a full and optimal training cycle.

\section{Limitations}

The test should be repeated in groups with larger sample sizes to determine exactly whether the group of women after CS without pain can be defined as a normative group, and to compare the results of abdominal muscle tests in various dysfunctions. The investigated groups were not homogenous, which could have exerted an impact on the study results. It would be interesting to compare the above results with a group of women after CS with pain in the lumbar and sacral spine.

\section{Conclusions}

Electromyographic parameters of fatigue of the abdominal muscles are similar in women after VD, women after CS, and women who have not given birth. CS in asymptomatic women does not affect the deterioration of abdominal muscle strength in the 15-second prone bridge test.

\section{Disclosure statement}

No author has any financial interest or received any financial benefit from this research.

\section{Conflict of interest}

The authors state no conflict of interest.

\section{References}

1. Gilleard WL, Brown JM. Structure and function of the abdominal muscles in primigravid subjects during pregnancy and the immediate postbirth period. Phys Ther. 1996;76(7):750-762; doi: 10.1093/ptj/76.7.750.

2. Benjamin DR, van de Water ATM, Peiris CL. Effects of exercise on diastasis of the rectus abdominis muscle in the antenatal and postnatal periods: a systematic review. Physiotherapy. 2014;100(1):1-8; doi: 10.1016/j.physio. 2013.08.005.

3. Gürşen C, İnanoğlu D, Kaya S, Akbayrak T, Baltacı G. Effects of exercise and Kinesio taping on abdominal recovery in women with cesarean section: a pilot randomized controlled trial. Arch Gynecol Obstet. 2016;293(3): 557-565; doi: 10.1007/s00404-015-3862-3.

4. Kanakaris NK, Roberts CS, Giannoudis PV. Pregnancyrelated pelvic girdle pain: an update. BMC Med. 2011;9: 15; doi: 10.1186/1741-7015-9-15.

5. Pel JJM, Spoor CW, Goossens RHM, Pool-Goudzwaard AL. Biomechanical model study of pelvic belt influence on muscle and ligament forces. J Biomech. 2008; 41(9):1878-1884; doi: 10.1016/j.jbiomech.2008.04.002.

6. Mens JMA, Pool-Goudzwaard A. The transverse abdominal muscle is excessively active during active straight leg raising in pregnancy-related posterior pelvic girdle pain: an observational study. BMC Musculoskelet Disord. 2017;18(1):372; doi: 10.1186/s12891-017-1732-9.

7. Fast A, Shapiro D, Ducommun EJ, Friedmann LW, Bouklas T, Floman Y. Low-back pain in pregnancy. Spine. 1987; 12(4):368-371; doi: 10.1097/00007632-19870500000011.

8. Kwon Y-J, Hyung E-J, Yang K-H, Lee H-O. How different modes of child delivery influence abdominal muscle activities in the active straight leg raise. J Phys Ther Sci. 2014;26(8):1271-1274; doi: 10.1589/jpts.26.1271.

9. Awad E, Ahmed H, Yousef A, Saab IM. Effect of antenatal exercise on mode of delivery in gestational diabetic females: a single-blind randomized controlled trial. Physiother Quart. 2019;27(2):1-5; doi: 10.5114/pq.2019.84270.

10. Cyriax JH. Cyriax: illustrated manual of orthopaedic medicine [in Polish]. Wrocław: Elsevier; 1996.

11. Kristiansson $P$, Svärdsudd K. Discriminatory power of tests applied in back pain during pregnancy. Spine. 1996; 21(20):2337-2343; doi: 10.1097/00007632-19961015000006.

12. Van der Wurff $P$, Hagmeijer RH, Meyne W. Clinical tests of the sacroiliac joint. A systematic methodological review. Part 1: reliability. Man Ther. 2000;5(1):30-36; doi: 10.1054/math. 1999.0228.

13. Workman JC, Docherty D, Parfrey KC, Behm DG. Influence of pelvis position on the activation of abdominal and hip flexor muscles. J Strength Cond Res. 2008;22(5): 1563-1569; doi: 10.1519/JSC.0b013e3181739981.

14. Callahan M. Comparison of muscle activation in the active straight leg raise and double straight leg lowering test. A thesis presented to the College of Graduate and Professional Studies. Department of Applied Medicine and Rehabilitation, Indiana State University; 2011. 
15. Oleksy Ł, Czarny W, Bajorek W, Król P, Mika A, Kielnar R. The evaluation of shoulder muscle fatigue in volleyball players. J Nov Physiother. 2018;8(2):388; doi: 10.4172/ 2165-7025.1000388.

16. Tong TK, Wu S, Nie J. Sport-specific endurance plank test for evaluation of global core muscle function. Phys Ther Sport. 2014;15(1):58-63; doi: 10.1016/j.ptsp.2013. 03.003.

17. De Blaiser C, De Ridder R, Willems T, Danneels L, Vanden Bossche L, Palmans T, et al. Evaluating abdominal core muscle fatigue: assessment of the validity and reliability of the prone bridging test. Scand J Med Sci Sports. 2018;28(2):391-399; doi: 10.1111/sms.12919.

18. Pereira LC, Botelho S, Marques J, Amorim CF, Lanza AH, Palma $P$, et al. Are transversus abdominis/oblique internal and pelvic floor muscles coactivated during pregnancy and postpartum? Neurourol Urodyn. 2013;32(5): 416-419; doi: 10.1002/nau.22315.

19. Norman E, Sherburn M, Osborne RH, Galea MP. An exercise and education program improves well-being of new mothers: a randomized controlled trial. Phys Ther. 2010;90(3):348-355; doi: 10.2522/ptj.20090139.

20. Morgen IM. Does caesarean section negatively influence the post-partum prognosis of low back pain and pelvic pain during pregnancy? Eur Spine J. 2007;16(1):115-121; doi: 10.1007/s00586-006-0098-8.

21. Parker MA, Millar LA, Dugan SA. Diastasis rectus abdominis and lumbo-pelvic pain and dysfunction - are they related? J Womens Health Phys Ther. 2009;33(2):15-22; doi: 10.1097/01274882-200933020-00003.

22. Boerma T, Ronsmans C, Melesse DY, Barros AJD, Barros FC, Juan L, et al. Global epidemiology of use of and disparities in caesarean sections. Lancet. 2018;392 (10155):1341-1348; doi: 10.1016/S0140-6736(18)31928-7.

23. Koroukian SM. Relative risk of postpartum complications in the Ohio Medicaid population: vaginal versus cesarean delivery. Med Care Res Rev. 2004;61(2):203224; doi: 10.1177/1077558703260123.

24. Blondeel N, Boeckx WD, Vanderstraeten GG, Lysens R, Van Landuyt K, Tonnard $\mathrm{P}$, et al. The fate of the oblique abdominal muscles after free TRAM flap surgery. $\mathrm{Br} \mathrm{J}$ Plast Surg. 1997;50(5):315-321; doi: 10.1016/s00071226(97)90539-7.

25. Davies GAL, Wolfe LA, Mottola MF, MacKinnon C. Joint SOGC/CSEP clinical practice guideline: exercise in pregnancy and the postpartum period. Can J Appl Physiol. 2003;28(3):330-341; doi: 10.1139/h03-024.

26. Guszkowska M. Antenatal anxiety and its determinants - a literature review [in Polish]. Perinatol Neonatol Ginekol. 2012;5(3):154-161.

27. Konopka J, Suchocki S, Puskarz R, Obst M. Caesarean section on demand without medical indications in patients' opinion [in Polish]. Perinatol Neonatol Ginekol. 2008;1(1):35-37.

28. Toporska A. The knowledge of women on healthy behaviours in the period of pregnancy and confinement [in Polish]. Chowanna. 2008;2:79-94.

29. Torbé D, Stolarek A, Lubkowska A, Torbé A. Physical activity recommended in the early postpartum period [in Polish]. Pomeranian J Life Sci. 2016;62(3):53-56; doi: 10.21164/pomjlifesci.182.

30. Mottola MF. Exercise in the postpartum period: practical applications. Curr Sports Med Rep. 2002;1(6):362-368; doi: 10.1249/00149619-200212000-00010. 